\title{
A Reassessment of Birth Defects for Children of Participants of the Air Force Health Study
}

\author{
George J. Knafl \\ Chapel Hill, NC, USA \\ Email: gknafl@outlook.com
}

How to cite this paper: Knafl, G.J. (2018) A Reassessment of Birth Defects for Children of Participants of the Air Force Health Study. Open Journal of Epidemiology, 8, 187-200.

https://doi.org/10.4236/ojepi.2018.84015

Received: September 4, 2018

Accepted: October 13, 2018

Published: October 16, 2018

Copyright $\odot 2018$ by author and Scientific Research Publishing Inc. This work is licensed under the Creative Commons Attribution International License (CC BY 4.0).

http://creativecommons.org/licenses/by/4.0/

\section{(c) (i) Open Access}

\begin{abstract}
The Air Force Health Study (AFHS), also called the Ranch Hand Study, investigated the impact of exposure to dioxin the toxic contaminant in Agent Orange on health, survival, and reproductive outcomes of male Air Force Vietnam War veterans. It was concluded that available reproductive outcome data did not provide support for an adverse association with paternal dioxin exposure. A more extensive set of AFHS data was used to reassess this conclusion, restricting to the case of birth defects in children fathered after the start of the first Vietnam War tour. Analyses started by repeating published analyses, followed by assessing decisions made in those analyses, for example, of excluding participants with dioxin levels below the detectable limit, using a threshold of 10 parts per trillion for a high dioxin level, and not adjusting for multiple conceptions/children of the same participant. Using data for all participants with measured dioxin levels, both veterans who served in Operation Ranch Hand and other non-Ranch Hand veterans, and after accounting for correlation within children of the same participant, the occurrence for children fathered after the start of the first tour of a major defect, a non-major defect, and multiple defects depended significantly on participants having a high dioxin level. These conclusions were not changed by consideration of covariates. In contrast to prior published analyses, the more extensive AFHS data provided support for an adverse effect of paternal dioxin exposure on birth defects. However, the study had many limitations that could have affected the conclusions.
\end{abstract}

\section{Keywords}

Birth Defects, Cross-Validation, Dioxin, Operation Ranch Hand

\section{Introduction}

The Air Force Health Study (AFHS), also called the Ranch Hand Study, was a 
longitudinal, prospective epidemiological study. It investigated the impact of herbicide exposure, especially to dioxin the toxic contaminant in Agent Orange, on the health, survival, and reproductive outcomes of male Air Force Vietnam War veterans [1] [2] [3]. Study participants included personnel responsible for herbicide handling and spraying in Vietnam as part of Operation Ranch Hand along with other veterans who served in Southeast Asia, but not necessarily in Vietnam.

Congress directed the Air Force to begin planning the study in 1979. Baseline data were collected in 1982 and follow-up data at five later time points up to 2002. Air Force personnel were eligible if they served as part of Operation Ranch Hand or if they served between 1962 and 1973 somewhere in Southeast Asia. Medical records were reviewed to verify reproductive outcomes. Participants' serum dioxin levels were also collected for use as a measure of herbicide exposure. By 1992, the medical record review had progressed to the point that an official report on reproductive outcomes was issued [3]. Medical records, including birth certificates, newborn clinic records, health records, and death certificates, were reviewed for all children, not just those self-reported by parents as having defects/disabilities, to avoid reporting bias. They also evaluated the possibility of differential reporting due to Ranch Hand participants seeking medical evaluation for birth defects more than the other veterans, thereby making their children's birth defects more verifiable, especially in births conceived after the start of the first tour. No evidence of such a verification bias was found. It was concluded that data verified by that time did not provide support for an adverse association of paternal dioxin exposure on reproductive outcomes. Results from the 1992 report were published in 1995 [4], drawing the same conclusion of no adverse association. See [5] for more details on the study.

Ranch Hand Study data were released in 2000 to the Vietnam Veterans of America with some secondary analysis results reported at a conference in 2003 [6]. These data were used to conduct a new set of secondary analyses. The objective of these latest analyses was to reassess the dependence of birth defects in the children of AFHS participants on dioxin exposure, starting from prior published AFHS analyses [4].

\section{Methods}

\subsection{Birth Defect Data}

Data were available for 6922 live-born children with known conception date (Table 1) that were fathered by 2613 AFHS participants (Table 2). Birth defects were verified through medical records and categorized into major, minor, and unclassified (Table 3). Dioxin levels were measured for 2038 (78.0\%) participants with values either ranging from 1.25 to 314.2 parts per trillion (ppt) or below the detection limit for 115 (5.6\%) of these participants (treated as $\leq 1 \mathrm{ppt}$ ).

The 1160 participants with measured dioxin levels fathered 2188 children after the start of their first tour of duty during the Vietnam War (Table 4). Their 
Table 1. Frequencies and percentages for live-born children by child conception type and participant dioxin type.

\begin{tabular}{cccc}
\hline Conception $^{\mathrm{a}}$ & $\mathrm{N}(\%)$ & Father's Dioxin & $\mathrm{N}(\%)^{\mathrm{c}}$ \\
\hline before & $4180(60.4)$ & measured & $3290(78.7)$ \\
& & missing & $890(21.4)$ \\
\multirow{2}{*}{ after } & $2742(39.6)$ & measured & $2188(79.8)$ \\
& & missing & $554(20.2)$ \\
either & $6922(100)$ & measured & $5478(79.1)$ \\
& & missing & $1444(20.9)$ \\
\hline
\end{tabular}

a: Relative to the start of the participant's first Vietnam War tour; b: Percentages for all children; c: Percentages for children within child type.

Table 2. Frequencies and percentages of participants by children's conception and participant's dioxin types.

\begin{tabular}{cccc}
\hline Conception $^{\mathrm{a}}$ & $\mathrm{N}(\%)^{\mathrm{b}}$ & Dioxin & $\mathrm{N}(\%)^{\mathrm{c}}$ \\
\hline some before & $1679(64.3)$ & measured & $1294(77.1)$ \\
& & missing & $385(22.9)$ \\
some after & $1497(57.3)$ & measured & $1160(77.5)$ \\
& & missing & $337(22.5)$ \\
some both & $553(21.5)$ & measured & $416(73.9)$ \\
& & missing & $147(26.1)$ \\
some either & $2613(100)$ & measured & $2038(78.0)$ \\
& & missing & $575(22.0)$ \\
\hline
\end{tabular}

a: Relative to the start of the participant's first Vietnam War tour; b: Percentages for all participants; c: Percentages for participants within children's conception type.

Table 3. Frequencies and percentages for live-born children by child conception type, participant dioxin type, and defect type.

\begin{tabular}{cccccc}
\hline & & \multicolumn{4}{c}{${\text { Defect Type N }(\%)^{\mathrm{b}}}^{\text {Fonception }}{ }^{\mathrm{a}}$} \\
& $\begin{array}{c}\text { Father's } \\
\text { Dioxin }\end{array}$ & None & Non-major & Major & Multiple \\
\hline before & measured & $2929(89.0)$ & $237(7.2)$ & $124(3.8)$ & $56(1.7)$ \\
& missing & $807(90.7)$ & $62(7.0)$ & $21(2.4)$ & $4(0.1)$ \\
\multirow{2}{*}{ after } & measured & $1720(78.6)$ & $336(15.4)$ & $132(6.0)$ & $79(3.6)$ \\
& missing & $481(86.8)$ & $51(9.2)$ & $22(4.0)$ & $16(2.9)$ \\
either & measured & $4649(84.9)$ & $573(10.5)$ & $256(4.7)$ & $135(2.5)$ \\
& missing & $1288(89.2)$ & $113(7.9)$ & $43(3.0)$ & $20(1.4)$ \\
\hline
\end{tabular}

a: Relative to the start of the participant's first Vietnam War tour; b: Percentages for children within child type and dioxin level type; c: Child has non-major (minor or unclassified) defects but no major defects.

detectable dioxin levels ranged from 1.33 to $314.2 \mathrm{ppt}$ plus 71 (6.1\%) others below the detectable limit; $7.0 \%$ were Black, $30.9 \%$ officers, and $40.4 \%$ flyers. 
Table 4. Descriptive statistics for live-born children conceived after the start of the first tour and for participants with measured dioxin levels.

\begin{tabular}{|c|c|c|c|}
\hline & Range & Median & $\mathrm{N}(\%)$ \\
\hline \multicolumn{4}{|l|}{$\begin{array}{l}\text { for } 2188 \text { live-born children conceived after the } \\
\text { start of the first tour }\end{array}$} \\
\hline mother smoked during pregnancy & & & $389(17.8)$ \\
\hline mother drank during pregnancy & & & $137(6.3)$ \\
\hline mother's age at birth of the child & $16-47$ & 27 & \\
\hline father's age at birth of the child & $21-59$ & 30 & \\
\hline \multicolumn{4}{|l|}{$\begin{array}{l}\text { for } 1160 \text { participants with measured dioxin } \\
\text { levels who fathered the above children }\end{array}$} \\
\hline black & & & $81(7.0)$ \\
\hline officer & & & $359(30.9)$ \\
\hline flyer & & & $469(40.4)$ \\
\hline Ranch Hand & & & $476(41.0)$ \\
\hline detectable dioxin level (ppt) & $1.33-314.2$ & 5.15 & \\
\hline undetected dioxin level & & & $71(6.1)$ \\
\hline
\end{tabular}

ppt-parts per trillion.

Mothers smoked for $17.8 \%$ of these pregnancies and drank for $6.3 \%$. Median age at birth for mothers was 27 years and 30 years for fathers. Only the mother's age had missing values with ages missing for $1.2 \%$ of the children.

\subsection{Published Analyses Conducted by the AFHS}

Wolfe et al. [4] reported results on reproductive outcome data available at that time for participants with measured dioxin levels. They considered only conceptions that occurred after the start of the first tour. They treated dioxin levels at or below $10 \mathrm{ppt}$ as background exposures. They excluded data for non-Ranch Hand participants with dioxin levels greater than $10 \mathrm{ppt}$ and categorized Ranch Hand participants into those with dioxin $\leq 10$ versus $>10$. They also excluded participants with dioxin levels below the detectable limit. This left data for 2241 pregnancies, 1773 children, and 1024 participants. Although many participants fathered multiple conceptions/children, these published analyses did not account for possible correlation within outcome values for conceptions/children of the same participant. The dioxin $>10$ category was further categorized on the basis of low/high values for an estimate of the dioxin exposure at the time of conception, but that issue was not considered here.

\subsection{Data Analysis}

Reported analyses started by rerunning published analyses using currently available data, but only for birth defects and not for other reproductive outcomes. Then, several sensitivity analyses were conducted including assessing the effect of excluding participants with dioxin values below the detectable limit, of 
excluding non-Ranch Hand participants with dioxin $>10$, of correlation within outcomes for children of the same participant, and of the choice of the threshold of 10 for determining background dioxin exposures. Possible differences for Ranch Hand and non-Ranch Hand participants and comparisons to birth defects for children conceived before the start of the tour were also addressed.

Analyses modeled the occurrence of birth defect outcomes using logistic regression models. To account for correlation within outcomes for children of the same participant, analyses used generalized estimating equations (GEE) techniques assuming an exchangeable correlation structure with the correlation the same for all pairs of children of the same participant. Three birth defect outcomes were considered: the occurrence of a major defect compared to a non-major (minor or unclassified) or no defect, of multiple $(>1)$ defects compared to one or no defect, and of a non-major defect compared to no defect after removing children born with a major defect. Wald $\chi^{2}$ tests for zero coefficients based on empirical standard error estimates were generated for these models. Standard $\chi^{2}$ tests were generated for analyses reproducing published results. Significance was set at $\mathrm{p}<0.05$.

Alternate models for the occurrence of the three birth defect outcomes were compared using adaptive regression methods [7]. Models were evaluated using 10-fold extended likelihood cross-validation $\left(\mathrm{LCV}^{+}\right)$scores based on the multivariate normal likelihood evaluated at Pearson residuals as defined and justified in [7]. The data were randomly partitioned into 10 disjoint subsets or folds. The extended likelihood for each fold was computed using the fold data and parameters estimated using data in the other folds. These deleted fold extended likelihoods were combined into an $\mathrm{LCV}^{+}$score with a higher value indicating a better model. Thresholds for high dioxin levels were adaptively identified using grid searches maximizing the $\mathrm{LCV}^{+}$score. These searches first considered alternative thresholds that were multiples of 0.5 , then multiples of 0.1 around the multiple of 0.5 generating the best $\mathrm{LCV}^{+}$score. Although generated thresholds maximized the $\mathrm{LCV}^{+}$score, the associated threshold effect was not guaranteed to be significant.

Reported analyses were generated using $\mathrm{SAS}^{\circledR}$ version 9.4 (SAS Institute, Inc., Cary, NC). SAS macros are available from the author for computing $\mathrm{LCV}^{+}$scores for GEE models (and more general regression models as well) and for searching through alternate thresholds for a high dioxin level. Code generating reported results as well as generated output are also available.

\section{Results}

\subsection{Rerunning Published Analyses Conducted by the Air Force Health Study}

Of the 2188 children born after the start of the first tour for fathers with measured dioxin levels (Table 5), excluding children for participants with dioxin levels below the detection limit removes 130 children (5.9\%). Excluding children 
Table 5. Frequencies and percentages for participants who fathered children after the start of the first tour as considered in published analyses.

\begin{tabular}{cccccc}
\hline \multicolumn{2}{c}{ Participants } & & \multicolumn{2}{c}{ Children } \\
\hline \multirow{2}{*}{ Ranch Hand } & Dioxin (ppt) & All & With exclusions & All & With exclusions \\
& N (\%) & N (\%) & N (\%) & N (\%) \\
\hline \multirow{2}{*}{ yes } & $1-10$ & $189(16.3)$ & $189(17.5)$ & $342(15.6)$ & $342(16.8)$ \\
& $>10$ & $281(24.2)$ & $281(26.1)$ & $555(25.4)$ & $555(27.3)$ \\
no & $1-10$ & $608(52.4)$ & $608(56.4)$ & $1135(51.9)$ & $1135(55.9)$ \\
& $>10$ & $11(9.5)$ & - & $26(1.2)$ & - \\
\multirow{2}{*}{ either } & undetected & $71(6.1)$ & - & $130(5.9)$ & - \\
& total & $1160(100)$ & $1078(100)$ & $2188(100)$ & $2032(100)$ \\
\hline
\end{tabular}

ppt-parts per trillion.

for non-Ranch Hand participants with dioxin $>10$ removes $26(1.2 \%)$ more children, leaving 2032 children (92.9\%). Of these children, 1135 (55.9\%) were born to non-Ranch Hand participants with $1<$ dioxin $\leq 10,342(16.8 \%)$ to Ranch Hand participants with $1<$ dioxin $\leq 10$, and 555 (27.3\%) to Ranch Hand participants with dioxin $>10$. Corresponding percentages for published analyses were similar at $55.3 \%, 16.0 \%$, and $28.7 \%$ out of 1773 children [4].

Of the 2032 children, 125 (6.2\%) had a major defect and 77 (3.8\%) had multiple defects. Of the remaining 1907 children without major defects, 316 (16.6\%) had a non-major (minor or unclassified) defect. Using standard $\chi^{2}$ tests, participant-dioxin exposure type (non-Ranch Hand, $1<$ dioxin $\leq 10$; Ranch Hand, $1<$ dioxin $\leq 10$; Ranch Hand, dioxin $>10$ ) was not significantly related to having a major defect $(\mathrm{p}=0.074)$, to having multiple defects $(\mathrm{p}=0.144)$, and to having a non-major defect compared to no defect $(\mathrm{p}=0.927)$. These conclusions were consistent with those reported by Wolfe et al. [4].

\subsection{Impact of Excluding Participants}

Including the 130 children for participants with dioxin levels below the detectable limit $(\leq 1)$ increases the number of children to 2162 with $129(6.0 \%)$ having a major defect and 79 (3.7\%) having multiple defects. Of the 2033 children without major defects, 334 (16.4\%) had a non-major defect.

Using standard $\chi^{2}$ tests, participant-dioxin exposure type (non-Ranch Hand, dioxin $\leq 10$; Ranch Hand, dioxin $\leq 10$; Ranch Hand, dioxin $>10$ ) was not significantly related to having multiple defects $(p=0.103)$ and to having a non-major defect compared to no defect ( $\mathrm{p}=0.943$ ), but was now significantly related to having a major defect $(\mathrm{p}=0.043)$. Children of Ranch Hand participants with dioxin $>10$ were more likely to have a major defect than children for non-Ranch Hand participants and Ranch Hand participants having dioxin $\leq 10$ (8.1\% versus $5.1 \%$ and $5.7 \%)$.

Of the 26 non-Ranch Hand participants with dioxin > 10, 3 (11.5\%) had a major defect. This result combined with the prior result suggested that the oc- 
currence of a major defect depended on having a high dioxin level for both Ranch Hand and non-Ranch Hand participants combined. This was supported: having dioxin $>10$ was significantly related to having a major defect $(p=0.009)$. Children for participants with dioxin $>10$ were more likely to have a major defect $(8.3 \%$ versus $5.2 \%$; odds ratio [OR] $1.63,95 \%$ confidence interval [CI] 1.13 2.36).

\subsection{Occurrence of Birth Defects in Children of Participants with Measured Dioxin Levels}

The 1160 participants with measured dioxin levels fathered 1 - 10 children after the start of the first tour. Of these participants, 1038 (89.5\%), $113(9.7 \%), 8$ $(0.7 \%)$, and $1(0.1 \%)$ had $0,1,2$, and 3 children with a major defect, respectively; 1083 (93.4\%), $75(6.5 \%)$, and $2(0.2 \%)$ had 0,1 , and 2 children with multiple defects ( 2 - 5 defects per child), respectively; 292 (25.2\%) had dioxin $>10$. These participants fathered 2,188 children after the start of the first tour with 132 (6.0\%) having a major defect and 79 (3.6\%) with multiple defects.

For the 1130 participants fathering children with non-major or no defects, 847 (75.0\%), 234 (20.7\%), $45(4.0 \%)$, and $4(0.4 \%)$ had $0,1,2$, and 3 children with a non-major defect, respectively; $282(25.0 \%)$ had dioxin $>10$. These participants fathered 2,056 children after the start of the first tour with 336 (16.3\%) having a non-major defect.

Using GEE techniques to account for within-participant correlation (as for all subsequent analyses), the occurrence of a major defect was significantly related to having dioxin $>10$ versus dioxin $\leq 10(\mathrm{p}=0.009$; estimated mean 0.083 versus 0.052; OR 1.65, 95\% CI 1.13 - 2.41) while the occurrence of multiple defects was non-significant $(\mathrm{p}=0.066)$. Excluding participants with children having major defects, the occurrence of a non-major defect compared to no defect was not significantly related to having dioxin $>10$ versus dioxin $\leq 10(\mathrm{p}=0.807)$.

\subsection{Adaptive Generation of Thresholds for a High Dioxin Level}

Table 6 presents results for adaptive selection of thresholds for a high dioxin level. Three outcomes were considered: the occurrence of a major defect versus a non-major or no defect, of multiple defects versus 1 or no defect, and of a non-major defect versus no defect.

A grid search identified that the threshold of 6.3 maximized the $\mathrm{LCV}^{+}$score for predicting the occurrence of a major defect. The occurrence of a major defect was significantly related to having dioxin $>6.3$ versus dioxin $\leq 6.3(\mathrm{p}<0.001)$ with estimated probability of a major defect 0.085 versus 0.045 .

A grid search identified that the threshold of 8.8 maximized the $\mathrm{LCV}^{+}$score for predicting the occurrence of multiple defects. The occurrence of multiple defects was significantly related to having dioxin $>8.8$ versus dioxin $\leq 8.8$ ( $\mathrm{p}=$ 0.012 ) with estimated probability of a multiple defects 0.052 versus 0.030 .

A grid search identified that the threshold of 3.7 maximized the $\mathrm{LCV}^{+}$score 
Table 6. Adaptive dioxin exposure thresholds for predicting birth defect outcomes for children of Air Force Health Study participants conceived after the start of the first Vietnam War tour.

\begin{tabular}{cccccccc}
\hline Defect Type & $\begin{array}{c}\text { Dioxin } \\
\text { (ppt) }\end{array}$ & $\begin{array}{c}\text { Participants } \\
\mathrm{N}(\%)\end{array}$ & $\begin{array}{c}\text { Children } \\
\mathrm{N}(\%)\end{array}$ & p-Value & OR & $95 \%$ CI & $\begin{array}{c}\text { Estimated } \\
\text { Probability }\end{array}$ \\
\hline $\begin{array}{c}\text { major } \\
\text { defect }^{\mathrm{a}}\end{array}$ & $>6.3$ & $444(38.3)$ & $855(39.1)$ & $<0.001$ & 1.97 & $1.36-2.85$ & 0.085 \\
& $\leq 6.3$ & $716(61.7)$ & $1333(60.9)$ & & & & 0.045 \\
$\begin{array}{c}\text { multiple } \\
\text { defects }^{\mathrm{a}}\end{array}$ & $>8.8$ & $324(27.9)$ & $639(29.2)$ & 0.014 & 1.78 & $1.12-2.82$ & 0.052 \\
& $\leq 8.8$ & $836(72.1)$ & $1549(70.8)$ & & & & 0.030 \\
$\begin{array}{c}\text { non-major } \\
\text { defect }\end{array}$ & $>3.7$ & $731(64.7)$ & $1359(66.1)$ & 0.034 & 1.34 & $1.02-1.76$ & 0.176 \\
& $\leq 3.7$ & $399(35.3)$ & $697(33.9)$ & & & & 0.137 \\
\hline
\end{tabular}

ppt-parts per trillion; a: For 1160 participants with measured dioxin levels who fathered 2188 live-born children after the start of their first tour; b: For 1130 participants with measured dioxin levels who fathered 2056 live-born children without a major defect after the start of their first tour.

for predicting the occurrence of a non-major defect (compared to no defects). The occurrence of a non-major defect was significantly related to having dioxin $>3.7$ versus dioxin $\leq 3.7(p=0.034)$ with estimated probability of a non-major defect 0.176 versus 0.137 .

\subsection{Differences for Ranch Hand Participants}

The AFHS surveyed non-Ranch Hand participants to constitute a comparison group to the Ranch Hand participants. The Ranch Hand participants do represent a high dioxin group with dioxin values ranging from undetected to 314.2 ppt with median $13.6 \mathrm{ppt}$ compared to values for non-Ranch hand participants ranging from undetected to $54.8 \mathrm{ppt}$ with median $3.6 \mathrm{ppt}$. However, Ranch Hand participants were not significantly more likely than non-Ranch Hand participants to have children with a major defect $(\mathrm{p}=0.059)$, with multiple defects $(\mathrm{p}=0.312)$, and with a non-major versus no defect $(\mathrm{p}=0.926)$. After controlling for a high dioxin level (varying with the outcome), being a Ranch Hand participant, and their interaction, the interaction had a non-significant effect on the occurrence of multiple defects $(p=0.907)$ and the occurrence of a non-major versus no defect $(\mathrm{p}=0.197)$. The interaction had a significant $(\mathrm{p}=$ 0.024 ) effect on the occurrence of a major defect, but the estimated probability of a major defect was larger for non-Ranch Hand participants with a high dioxin level than Ranch Hand participants with a high dioxin level (0.129 versus 0.075).

\subsection{Impact of Missing Dioxin Levels}

Of 1497 participants who fathered 2742 children after the start of their tours, dioxin levels were missing for 337 (22.5\%) participants who fathered 554 (20.3\%) children after the start of the first tour (Table $1 \&$ Table 2). Data for 
these participants were combined with data for participants with measured dioxin levels to assess the impact of having a missing dioxin level on birth defect outcomes.

The participant having a missing dioxin level had a non-significant effect on the occurrence of a major defect $(\mathrm{p}=0.060)$ and on the occurrence of multiple defects $(p=0.402)$, but had a significant effect on the occurrence of a non-major versus no defect $(\mathrm{p}<0.001)$. The estimated probability of having a non-major versus no defect was lower for children fathered by participants with a missing dioxin level (0.097 compared to 0.163).

\subsection{Comparison to Children Conceived Prior to the Start of the First Tour for Participants with Measured Dioxin Levels}

A total of 5478 children were fathered either before or after the start of the first tour by 2038 participants with measured dioxin levels. These participants fathered from 1 - 11 children. Models were considered for birth defect outcomes for these children including two effects: 1) being conceived after the start of the first tour for participants having a high dioxin level (adjusted for the outcome) and; 2) being conceived before the start of the first tour. In this way, birth defect outcomes for children conceived before the start of the first tour are compared to birth defect outcomes for children conceived after the start of the first tour for participants having a low/background dioxin level.

The estimated probability of a child having a major defect when born before the start of the first tour was not significantly different from the estimated probability of a child having a major defect when born after the start of the first tour for a participant having a low/background dioxin level $(p=0.283)$. The estimated probability of a child having multiple defects when born before the start of the first tour was significantly lower than the estimated probability of a child having a major defect when born after the start of the first tour for a participant having a low/background dioxin level $(\mathrm{p}=0.005$, estimated probability 0.017 versus 0.030 ). In both cases, the effect to being conceived after the start of the first tour for participants having a high dioxin level remained significant $(\mathrm{p}<$ $0.05)$ with larger estimated probability.

A total of 5222 children without a major defect were fathered either before or after the start of the first tour by 2017 participants with measured dioxin levels. These participants fathered from 1 - 11 children. The estimated probability of a child having a non-major defect was significantly lower when born before the start of the first tour than when born after the start of the first tour for a participant having a low/background dioxin level $(\mathrm{p}<0.001$, estimated probability 0.077 versus 0.138 ). The effect to being conceived after the start of the first tour for participants having a high dioxin level remained significant $(\mathrm{p}<0.05)$ with larger estimated probability.

\subsection{Adjusting for Covariates}

Wolfe et al. [4] considered seven covariates including whether the mother 
smoked or not and drank or not during the pregnancy, the age of the mother and of the father at the birth of the child, and whether the father was Black or not, an officer or not, and a flyer or not. See Table 4 for descriptive statistics for these covariates.

Covariate analyses were conducted for the three birth defect outcomes considered earlier for children fathered after the start of the tour by participants with measured dioxin levels. To assess the impact of a covariate on the conclusion of an effect to a high dioxin level on each outcome, the covariate was added to the model for that outcome based on the associated indicator for a high dioxin level.

After controlling for the effect of a high dioxin level $(>6.3)$, the occurrence of a major defect did not depend significantly $(p>0.05)$ on each of five covariates separately while the effect of a high dioxin level remained significant $(\mathrm{p}<0.001)$. The two exceptions with a significant effect were for the participant age at birth $(\mathrm{p}=0.013)$ and for Black participants $(0.039)$, but in both cases the effect to a high dioxin level remained significant $(\mathrm{p}<0.001)$. Further, when both of these two covariate effects were included in the model, both remained significant (increasing with participant age, $\mathrm{p}=0.014$; decreasing for Black participants, $\mathrm{p}=$ $0.039)$ as also did the effect of a high dioxin level $(\mathrm{p}<0.001)$.

After controlling for the effect of a high dioxin level $(>8.8)$, the occurrence of multiple defects did not depend significantly $(\mathrm{p}>0.05)$ on each of the seven covariates separately. The effect of a high dioxin level remained significant ( $\mathrm{p}<$ $0.05)$ in all cases.

After controlling for the effect of a high dioxin level $(>3.7)$, the occurrence of a non-major versus no defect did not depend significantly $(p>0.05)$ on each of the seven covariates separately. The effect of a high dioxin level remained significant $(\mathrm{p}<0.05)$ in all cases.

\section{Discussion}

Three birth defect outcomes were considered: having a major defect versus a non-major (minor or unclassified) or no defect, having multiple defects $(>1)$ versus one or no defect, and, after removing children with a major defect, having a non-major defect versus no defect. When published analyses [4] were rerun addressing the occurrence of these three birth defect outcomes in children fathered after the start of the first tour using the more extensive AFHS data set, the same conclusion of no significant effect to participant-dioxin exposure type (non-Ranch Hand, $1<$ dioxin $\leq 10$; Ranch Hand, $1<$ dioxin $\leq 10$; Ranch Hand, dioxin $>10$ ) held in all three cases. After including birth defect data for participants with dioxin values below the detectable limit $(\leq 1)$, the effect of participant-dioxin exposure type on the occurrence of multiple defects and of a non-major defect remained non-significant, but its effect on the occurrence of a major defect became significant. The proportion of children with a major defect for non-Ranch Hand participants with dioxin $>10$ was consistent with the proportion of children with a major defect for Ranch Hand participants with dioxin $>10$. Conse- 
quently, subsequent analyses included these data for non-Ranch Hand participants and considered the effect of a high dioxin level without distinguishing between Ranch Hand and non-Ranch Hand participants (except when addressing this effect as a possible covariate).

AFHS participants with measured dioxin levels fathered from 1 - 10 children after the start of the first tour. The occurrence of birth defects in children for the same father would likely be correlated, so prior reported analyses were limited by not adjusting for this issue as were prior published analyses. This limitation was avoided by modeling correlations between birth defect outcomes for pairs of children for the same participant using GEE techniques. After adjusting for this correlation, having a high dioxin level ( $>10$, as used in published analyses) was significantly related to an increased probability of a major defect but not of multiple defects or of a non-major defect.

Using adaptive methods [7] (Table 6), alternate thresholds for a high dioxin level were identified for modeling the three birth defect outcomes for children conceived after the start of the first Vietnam War tour. Having a high dioxin level was significantly related to a larger estimated probability for children conceived after the start of the first tour in all three cases of having a major defect (dioxin > 6.3), multiple defects (dioxin > 8.8), and a non-major defect (dioxin > 3.7). Consequently, the conclusion of no effect to dioxin on birth defect outcomes in published analyses was a consequence of the combination of using a smaller data set, excluding selected sets of children, not adjusting for correlation within children of the same participant, and most importantly using a fixed level as a threshold for a high dioxin level (dioxin >10). The opposite conclusion of an adverse effect of paternal dioxin exposure on birth defects held when these limitations were resolved as well as after controlling for the same covariates considered in prior published analyses [4].

Sensitivity analyses suggested that it was not necessary to distinguish between Ranch Hand and non-Ranch Hand participants. This suggests that identified adverse effects to high dioxin levels are reasonably treated as holding equally for both groups of participants.

Children conceived before the start of the first tour for all participants with measured dioxin levels were not significantly less likely to have a major defect then children conceived after that for participants with low/background dioxin levels, but were significantly less likely to have multiple defects or to have non-major defect. In all three cases, children conceived after the start of the first tour to participants with high dioxin levels were still significantly more likely to have the associated birth defect type. Consequently, children born before the start of the first tour were less likely to have two of the three birth defect outcomes than children born after that, indicating that background levels of these outcomes increased from before to after the Vietnam War, but high dioxin levels still had an adverse effect on all three outcomes for children conceived after the start of the first tour for participants with high dioxin levels. 


\subsection{Limitations}

Dioxin levels were not measured for substantial numbers of AFHS participants; results might have changed if these dioxin levels had been measured. However, the occurrence of a major defect and of multiple defects was not significantly different for children of participants with missing versus measured dioxin levels, suggesting missing dioxin levels may not have biased results for these two more serious birth defect outcomes. On the other hand, the occurrence of a non-major defect was significantly lower for children of participants with missing dioxin levels compared to participants with measured dioxin levels. This suggests that missing dioxin levels may have biased results for this less serious birth defect outcome unless the actual dioxin levels for these participants were mainly low levels.

Analyses were based on post-exposure serum dioxin levels measured well after participants' tours ended and should be interpreted with this in mind. In particular, selected thresholds for adverse dioxin exposure were most likely smaller due to decay than if exposure had been measured just after the last tour. However, the issue of decay was not addressed in reported analyses as well as the related issue of varying times between the end of the last tour and conceptions. The possible effect of the combined length of all Vietnam War tours was also not addressed. In any case, as would be expected, the thresholds identified for the three birth defect outcomes increased with the severity of the birth defect type (i.e., 3.7 for as non-major defect, 6.3 for as major defect, and 8.8 for multiple defects).

Reported analyses only addressed three birth defect outcomes. Other reproductive outcomes were available for live-born children, including categories of birth defects (e.g., affecting the respiratory system), developmental disabilities (e.g., hyperkinetic), and birth weight and also for all conceptions (e.g., spontaneous abortion). Future research is needed to address possible adverse dioxin effects on these other outcomes.

This was a secondary analysis of data from a previously conducted study. What can be addressed was limited by what information was available in those data. For example, the data did not provide information about participants' occupations after service in the Vietnam War, which could account for possible exposures after the end of the last tour. Information was also not available on whether spouses/partners also served in the Vietnam War, which given the results of Kang et al. [8] would be useful for separating maternal from paternal dioxin exposure. It was also possible that Air Force veterans with children having birth defects were more likely to agree to participate in the study. Also, data were only available for Air Force veterans, not for veterans of the other armed services, and so results may not generalize to all Vietnam veterans. However, the fact that results could reasonably be treated as holding equally for non-Ranch Hand versus Ranch Hand participant suggests that adverse effects might also hold for Vietnam War veterans of the other US armed services whose exposures 
would likely be similar to exposures for non-Ranch Hand participants.

Prior evidence for adverse dioxin exposure association with birth defects is limited. The CDC Vietnam Experience Study [9] found a significant increase (OR 1.3, 95\% CI: 1.2 - 1.4; controlling for seven other predictors) in defects for children of Vietnam veterans compared to children of non-Vietnam veterans. Aschengrau and Monson [10] found an increased, but non-significant level (OR 1.3, 95\% CI: 0.9 - 1.9) of defects in children of Vietnam veterans compared to children of men without known military service. Ngo et al. [11] concluded on the basis of a meta-analysis that parental exposure to dioxin appears to be associated with increased risk for birth defects. However, Fraser [12] questioned the validity of this meta-analysis. In contrast, the results of the current study provide novel substantive support for an adverse association of birth defects with paternal dioxin exposure. Furthermore, such results are plausible since Mattison [13] concluded on the basis of a literature review that offspring are vulnerable to all endpoints of developmental toxicity, including structural and functional abnormalities, as a consequence of paternal, and not just maternal, exposures to chemicals.

\subsection{Summary}

In conclusion, in contrast to prior published analyses of data from the AFHS, support was identified for an adverse effect to paternal dioxin exposure on birth defects in the children fathered by male Air Force Vietnam veterans after the start of their first Vietnam War tour. This opposite conclusion was the combined result of using a larger data set, not excluding selected participants, adjusting for correlation between outcomes for children of the same participant, and using a threshold for a high dioxin exposure level adaptively set separately for each birth defect outcome. These conclusions still held after adjusting for the same covariates considered in prior published analyses. However, the study had many limitations that could have affected these conclusions.

\section{Acknowledgements}

The data were provided by the Vietnam Veterans of America, which funded earlier analyses of these data, but the work reported here was unfunded.

\section{Conflicts of Interest}

The author declares no conflicts of interest regarding the publication of this paper.

\section{References}

[1] Wolfe, W.H., Michalek, J.E., Miner, J.C., Rahe, A.J., Silva, J., Thomas, W.F., Grubbs, W., Lustig, M.B., Karrison, T.G., Roegner, R.H. and Williams, D.E. (1990) Health Status of Air Force Veterans Occupationally Exposed to Herbicides in Vietnam: I. Physical Health. Journal of the American Medical Association, 264, 1824-1831. https://doi.org/10.1001/jama.1990.03450140046032 
[2] Michalek, J.E., Wolfe, W.H. and Miner, J.C. (1990) Health Status of Air Force Veterans Occupationally Exposed to Herbicides in Vietnam: II. Mortality. Journal of the American Medical Association, 264, 1832-1836. https://doi.org/10.1001/jama.1990.03450140054033

[3] Wolfe, W.H., Michalek, J.E., Miner, J.C. and Rahe, A.J. (1992) An Epidemiological Investigation of Health Effects in Air Force Personnel Following Exposure to Herbicides: Extract, Reproductive Outcomes, Executive Summary, Introduction and Conclusions. Armstrong Laboratory, Brooks Air Force Base, San Antonio, TX.

[4] Wolfe, W.H., Michalek, J.E., Miner, J.C., Rahe, A.J., Moore, C.A., Needham, L.L. and Patterson Jr., D.G. (1995) Paternal Serum Dioxin and Reproductive Outcomes among Veterans of Operation Ranch Hand. Epidemiology, 6, 17-22.

https://doi.org/10.1097/00001648-199501000-00005

[5] The Air Force Health Study Assets Research Program. https://www.ncbi.nlm.nih.gov/books/NBK286036/

[6] Knafl, G.J. and Schwartz, L.S. (2003) A Fresh Look at the Ranch Hand Data: Composite Birth Defects and Developmental Disabilities. Organohalogen Compounds, 64, 152-157.

[7] Knafl, G.J. and Ding, K. (2016) Adaptive Regression for Modeling Nonlinear Relationships. Springer International Publishing, Switzerland.

https://doi.org/10.1007/978-3-319-33946-7

[8] Kang, H.K., Mahan, C.M., Lee, K.Y., Magee, C.A., Mather, S.H. and Matanoski, G. (2000) Pregnancy Outcomes among US Women Vietnam Veterans. American Journal of Industrial Medicine, 38, 447-454. https://doi.org/10.1002/1097-0274(200010)38:4<447::AID-AJIM11>3.0.CO;2-J

[9] Centers for Disease Control (1989) Health Status of Vietnam Veterans: Volume V Reproductive Outcomes and Child Health. U.S. Department of Health and Human Services, Atlanta.

https://www.cdc.gov/nceh/veterans/pdfs/volumev/reproductiveoutcomesandchildh ealth2_9.pdf

[10] Aschengrau, A. and Monson, R.R. (1990) Paternal Military Service in Vietnam and the Risk of Late Adverse Pregnancy Outcomes. American Journal of Public Health, 80, 1218-1224. https://doi.org/10.2105/AJPH.80.10.1218

[11] Ngo, A.D., Taylor, R., Roberts, C.L. and Nguyen, T.V. (2009) Association between Agent Orange and Birth Defects: Systematic Review and Meta-Analysis. International Journal of Epidemiology, 35, 1220-1230. https://doi.org/10.1093/ije/dyl038

[12] Fraser, F.C. (2009) Does Paternal Exposure to Agent Orange Cause Birth Defects? American Journal of Medical Genetics Part A, 149A, 835-836. https://doi.org/10.1002/ajmg.a.32798

[13] Mattison, D.R. (2010) Environmental Exposures and Development. Current Opinions in Pediatrics, 22, 208-218. https://doi.org/10.1097/MOP.0b013e32833779bf 